Purpose Office workers commonly suffer from work-related musculoskeletal disorders associated with intensive computer use, and both the physical and mental workload are important risk factors. This study used a typing task to investigate how externally induced stress modulated heart rate variability and muscle activation. The results would inform the possible physical and mental interactions in work-related musculoskeletal disorders. Health care providers need to understand how individuals would respond to stressful task demands which are useful for designing intervention for patients with workrelated musculoskeletal disorders.

Methods Twenty three healthy adults were recruited by convenience sampling, with 11 males and 12 females (mean age $=22.3 \pm 2.4$ years; body mass index $=20.4 \pm 12.7$ ). All participants must be computer users for at least $4 \mathrm{~h} /$ day. Those with present or past history of cardiac disease, hypertension, neck and shoulder pain or injury in the past 12 month were excluded. Ethical approval was received from the Human Research Ethics Committee of the Hong Kong Polytechnic University. Each participant performed typing task using a standardised computer workstation in a quiet and environmental control room with an adjustable chair and a stable desk. The stroop-effect words (name of the colour and colour of the word) (CWT) were used for inducing the stress. There were five task conditions organised in increasing physical and/or mental demands: (1) self-paced copying, (2) fast copying, (3) fast copying CWT, (4) verbal CWT and (5) prolonged CWT. Each task was performed for 5 min with 5 min rest except task (5) with 20 min and rest (5) at 10 min. Task (2) exerted the most physical stress and task (3) showed higher mental and physical stress whereas task (5) induced the highest level of physical and mental stress. Heart rate variability (HRV) data was recorded using the Polar heart rate monitor Polar Electro, Finland). Simultaneous surface electromyography (EMG) data were collected from bilateral cervical erector spinae and upper trapezii muscles the Noraxon Telemyo 2400T G2 System (Noraxon, USA Inc. USA) during all five task and rest periods. After each task and rest, participants were asked to rate their perceived stress level. The R-R interval values (ms) were computed from raw signal curves in the software and exported as text files for further processing. $R-R$ intervals and spectrum analysis of HRV was performed in fast fourier transformation (FFT) using the a HRV analysis software version 11.1.0 (Nevrokard, Slovenia). Low frequency (LF 0.04-0.15 Hz), high frequency (HF 0.15-0.40 Hz), LF/HF ratio and total power (normalised units, nu) were processed for data analysis. HRV was expressed as the low frequency to high frequency ratio (LF/HF ratio). EMG amplitudes were processed as root mean square (EMG-rms) and median (50th\%) amplitudes were computed.

Results Repeated measures ANOVA was performed to compare differences among the five tasks and recovery periods for the outcome variables of HRV, EMG amplitudes and perceived stress. Paired t-test was conducted to compare the change among five tasks. Changes in HRV for LF, HF, LF/HF ratio and total power correlated with task demands with significantly differed among the tasks $(p<0.05)$. Task (3) and task (5) were elicited higher response on LF/HF ratio. The muscle activity amplitudes of bilateral UT and CES increased significantly in tasks (2), (3) and (5). For perceived stress, the participants showed significant increases in 'tension' and 'pressure', but not in 'anxiety' and 'unease'. HRV, muscle activities, and perceived stress have recovered nearly to the baseline after all five rest periods.

Wang Yuling, Liang Qi, ${ }^{1}$ Grace Szeto, ${ }^{2}$ Chetwyn Chan ${ }^{2}$ The First Affiliated Hospital of Sun Yat-Sen University, Guangdong, China; ${ }^{2 T h e ~ H o n g ~ K o n g ~ P o l y t e c h n i c ~ U n i v e r s i t y, ~}$ Hong Kong, China

\title{
EFFECTS ON CARDIOVASCULAR RESPONSE, PERCEIVED STRESS AND CERVICAL MUSCLE ACTIVATION DURING PHYSICAL AND MENTAL CONDITIONS IN COMPUTER USERS
}


Conclusion The results showed that both physical and mental workloads produced significant increases in heart rate variability and cervical muscle activity. The participants also reported increased level of perceived tension and pressure. It is not known whether individuals with past history of musculoskeletal pain would respond differently during these similar task demands. This will be investigated in the future study. The results provide better understanding of how individuals respond to the influence of physical and mental stress factors which should be addressed in physiotherapy management of cardiovascular responses and musculoskeletal disorders during higher physical and/or mental workload. 